\title{
On the $q$-Monotonicity Preservation of Durrmeyer-Type Operators
}

\author{
Ulrich Abel@), Dany Leviatan and Ioan Raşa
}

Dedicated to our friend and colleague Professor Francesco Altomare on the occasion of his 70th birthday.

\begin{abstract}
We prove that various Durrmeyer-type operators preserve $q$-monotonicity in $[0,1]$ or $[0, \infty)$ as the case may be. Recall that a 1 -monotone function is nondecreasing, a 2-monotone one is convex, and for $q>2$, a $q$-monotone function possesses a convex $(q-2)$ nd derivative in the interior of the interval. The operators are the Durrmeyer versions of Bernstein (including genuine Bernstein-Durrmeyer), Szász and Baskakov operators. As a byproduct we have a new type of characterization of continuous $q$-monotone functions by the behavior of the integrals of the function with respect to measures that are related to the fundamental polynomials of the operators.
\end{abstract}

Mathematics Subject Classification. Primary 41A10, Secondary 41A29, $41 \mathrm{~A} 36$.

Keywords. Bernstein-Durrmeyer polynomials, Szász-Durrmeyer and Baskakov-Durrmeyer operators, $q$-monotone functions.

\section{Introduction}

Let $I$ be an interval on the real line, finite or infinite, open or closed or half open. For a function $f$, defined on $I$, denote

$$
\Delta_{h}^{1} f(x):=\Delta_{h} f(x):= \begin{cases}f(x+h)-f(x), & x, x+h \in I, \\ 0, & \text { otherwise, }\end{cases}
$$

and for $q \geq 1$,

$$
\Delta_{h}^{q+1} f(x):=\Delta_{h}^{q}\left(\Delta_{h} f(x)\right) .
$$

That is,

$$
\Delta_{h}^{q} f(x)= \begin{cases}\sum_{i=0}^{q}(-1)^{q-i}\left(\begin{array}{l}
q \\
i
\end{array}\right) f(x+i h), & x, x+q h \in I, \\
0, & \text { otherwise. }\end{cases}
$$


A function $f$ defined on $I$, is called $q$-monotone there, if $\Delta_{h}^{q} f(x) \geq 0, x \in I$, for all $h>0$. In particular a 1-monotone function is nondecreasing and a 2 -monotone one is convex. It is well-known (it goes back to T. Popoviciu, Mathematica 8 (1934), and R. P. Boas Jr. and D. V. Widder, Duke Math. J. 7 (1940)) that for $q>2, f$ is $q$-monotone in $I$, implies that $f$ possesses a convex $(q-2)$ nd derivative in the interior of $I$.

The classical Bernstein polynomials associated with a function $f$ which is defined on $[0,1]$, are defined by,

$$
\left(B_{n} f\right)(x):=\sum_{j=0}^{n} p_{n, j}(x) f\left(\frac{j}{n}\right), \quad x \in[0,1], \quad n \geq 1,
$$

where

$$
p_{n, j}(x):=\left(\begin{array}{c}
n \\
j
\end{array}\right) x^{j}(1-x)^{n-j}, \quad 0 \leq j \leq n .
$$

For the sake of simplicity we also denote $p_{0,0}(x) \equiv 1$ and $p_{n, j}(x) \equiv 0, j<0$ and $j>n$.

It is well-known (see, e.g., $[11,1.4(2)]$ ) that the Bernstein polynomials preserve $q$-monotonicity of all orders $q \geq 1$.

Durrmeyer [6] and, independently, Lupaş [12] have modified the Bernstein polynomials and defined what we call the Bernstein-Durrmeyer polynomials. Namely (see [4]), for an integrable $f$ on $[0,1]$, we set

$$
\left(D_{n} f\right)(x):=(n+1) \sum_{j=0}^{n} p_{n, j}(x) \int_{0}^{1} p_{n, j}(t) f(t) d t, \quad x \in[0,1], \quad n \geq 0 .
$$

It is well-known that if $f \in C[0,1]$, then the Bernstein-Durrmeyer polynomials uniformly approximate $f$ in $[0,1]$, as $n \rightarrow \infty$. As operators they are contractions in $L_{p}[0,1] 1 \leq p \leq \infty$ (where by $L_{\infty}[0,1]$ we mean $C[0,1]$ ), and are self-adjoint in $L_{2}[0,1]$. The interested reader may see details in [4] (see also [5]). We are interested in something else, namely, preservation of $q$-monotonicity.

Adell and de la Cal [2] proved that the Bernstein-Durrmeyer polynomials preserve monotonicity and convexity, applying techniques from probability and stochastic processes. In 2007 Attalienti and Raşa [3] proved that the Bernstein-Durrmeyer polynomials preserve $q$-monotonicity for all $q \geq 1$, using the heavy machinery of Karlin's Total Positivity [10]. In Section 2, we will show by elementary means that the Bernstein-Durrmeyer polynomials preserve $q$-monotonicity for all $q \geq 1$. We will use the proof to prove that the genuine Bernstein-Durrmeyer polynomials [8] (see also [7]) have the same property.

In Section 3, we discuss the Szász-Durrmeyer operators [13] and in Section 4 the Baskakov-Durrmeyer operators [15]. We will prove that both preserve $q$-monotonicity. For functions differentiable sufficiently many times and all required integrals converge, the $q$-monotonicity of the Szász-Durrmeyer operators was proved by Păltănea [14] (see [9] for details and other operators). Finally, in Section 5 we present some Raşa-type inequalities for Durrmeyertype operators. 


\section{2. $q$-monotonicity of the ordinary and genuine Bernstein-Durrmeyer polynomials}

We first prove the following

Theorem 2.1. Let $q \geq 1$, and assume that $f \in C[0,1]$ is q-monotone. Then $D_{n} f, n \geq 1$, is q-monotone.

A crucial lemma is

Lemma 2.2. Given $q \geq 1$, let $f \in C[0,1]$ be $q$-monotone. Then, for every $n \geq q$ and $0 \leq k \leq n-q$, we have

$$
\int_{0}^{1}\left[\sum_{i=0}^{q}(-1)^{q-i}\left(\begin{array}{l}
q \\
i
\end{array}\right) p_{n, k+i}(t)\right] f(t) d t \geq 0 .
$$

An immediate consequence of Lemma 2.2 and the proof of Theorem 2.1 is a new type of characterization of continuous $q$-monotone functions in $[0,1]$, which may be interesting by itself.

Corollary 2.3. Let $q \geq 1$ and assume that $f \in C[0,1]$. Then $f$ is $q$-monotone in $[0,1]$, if and only if, (2.1) holds for every $n \geq q$ and $0 \leq k \leq n-q$.

Proof. If $f \in C[0,1]$ is $q$-monotone, then (2.1) follows by Lemma 2.2. Conversely, if (2.1) is valid for every $n \geq q$ and $0 \leq k \leq n-q$, then by the proof of Theorem 2.1 it follows that $D_{n} f, n \geq 1$, is $q$-monotone. It is well-known (see, e.g., [4, Théorème II.2]) that $D_{n} f$ converges (uniformly) to $f$ in $[0,1]$, so it follows that $f$ is $q$-monotone.

Proof of Lemma 2.2. First, we observe that every $q$-monotone function $f \in$ $C[0,1]$ may be uniformly approximated by $q$ times continuously differentiable $q$-monotone functions on $[0,1]$. For example, one may take the Bernstein polynomials associated with $f$. Hence, in order to prove (2.1) it suffices to assume that $f \in C^{q}[0,1]$ so that by assumption $f^{(q)}(x) \geq 0, x \in[0,1]$.

Denote

$$
a_{n, k}:=\int_{0}^{1} p_{n, k}(t) f(t) d t
$$

and

$$
\Delta^{q} a_{n, k}:=\sum_{i=0}^{q}(-1)^{q-i}\left(\begin{array}{l}
q \\
i
\end{array}\right) a_{n, k+i}
$$

We will show that if $f \in C^{q}[0,1]$, then

$$
\Delta^{q} a_{n, k}=\frac{n !}{(n+q) !} \int_{0}^{1} p_{n+q, k+q}(t) f^{(q)}(t) d t, \quad 0 \leq k \leq n-q .
$$

To this end, straightforward differentiation yields that for any $m, l \geq 0$,

$$
p_{m+1, l+1}^{\prime}(x)=(m+1)\left(p_{m, l}(x)-p_{m, l+1}(x)\right) .
$$

Hence, it readily follows that

$$
\Delta^{1} a_{n, k}=\frac{1}{n+1} \int_{0}^{1} p_{n+1, k+1}(t) f^{\prime}(t) d t, \quad 0 \leq k \leq n-1 .
$$


Proceeding by induction, assuming (2.3) for $q-1$, and applying (2.4), we obtain (2.3) for $q$. Since $f^{(q)}(x) \geq 0$, our proof is complete.

Proof of Theorem 2.1. $D_{n} f$ is a polynomial of degree $n$. Thus, for $0 \leq n<q$ it is clearly $q$-monotone.

We write

$$
\left(D_{n} f\right)(x)=(n+1) \sum_{k=0}^{n} p_{n, k}(x) a_{n, k}, \quad 0 \leq x \leq 1,
$$

where $a_{n, k}$ is defined in (2.2).

Repeating, verbatim, the well-known computations for the Bernstein polynomials (see, e.g., $[11,1.4(2)]$ ), we have, for $n \geq q$,

$$
\left(D_{n} f\right)^{(q)}(x)=\frac{(n+1) !}{(n-q) !} \sum_{k=0}^{n-q} p_{n-q, k}(x) \Delta^{q} a_{n, k}, \quad 0 \leq x \leq 1 .
$$

Since by Lemma $2.2 \Delta^{q} a_{n, k} \geq 0$, for all $0 \leq k \leq n-q, n \geq q$, our proof is complete.

The genuine Bernstein-Durrmeyer polynomials associated with a function $f \in C[0,1]$, were defined by

$$
\begin{aligned}
\left(U_{n} f\right)(x):= & f(0) p_{n, 0}(x)+f(1) p_{n, n}(x) \\
& +(n-1) \sum_{k=1}^{n-1} p_{n, k}(x) \int_{0}^{1} p_{n-2, k-1}(t) f(t) d t, \quad x \in[0,1], \quad n \geq 2 .
\end{aligned}
$$

Recall that the advantage of the genuine Bernstein-Durrmeyer polynomials is that they preserve linear functions while the ordinary Bernstein-Durrmeyer polynomials only preserve constants.

We have the following.

Theorem 2.4. Let $q \geq 1$, and assume that $f \in C[0,1]$ is $q$-monotone. Then $U_{n} f, n \geq 2$, is q-monotone.

Proof. We have to separate the proof for $q=1$ and for $q \geq 2$, and we begin with the latter. Thus assume that $q \geq 2$. If $2 \leq n<q$, then there is nothing to prove. Thus, we assume that $n \geq q$.

Let $L$ be the linear function interpolating $f$ at $x=0,1$. Then, $g(x):=$ $(f-L)(x)$ vanishes at both $x=0,1$ and, if $f$ is $q$-monotone, so is $g$. Since $U_{n} g=U_{n} f-L$, if $U_{n} g$ is $q$-monotone, so is $U_{n} f$. Hence, without loss of generality, we may assume that $f(0)=f(1)=0$, so that

$$
\begin{aligned}
\left(U_{n} f\right)(x) & =(n-1) \sum_{k=1}^{n-1} p_{n, k}(x) \int_{0}^{1} p_{n-2, k-1}(t) f(t) d t \\
& =(n-1) \sum_{k=1}^{n-1} p_{n, k}(x) a_{n-2, k-1}, \quad x \in[0,1], \quad n \geq 2,
\end{aligned}
$$

where $a_{n, k}$ were defined in (2.2). 

rewrite

Denote $b_{n, k}:=a_{n-2, k-1}, 1 \leq k \leq n-1$, and $b_{n, 0}=b_{n, n}=0$. Then we

$$
\left(U_{n} f\right)(x)=(n-1) \sum_{k=0}^{n} p_{n, k}(x) b_{n, k} .
$$

As in (2.6) we have

$$
\left(U_{n} f\right)^{(q)}(x)=(n-1) \frac{n !}{(n-q) !} \sum_{k=0}^{n-q} p_{n-q, k}(x) \Delta^{q} b_{n, k} .
$$

If $n \geq q+2$, then by virtue of Lemma $2.2, \Delta^{q} b_{n, k}=\Delta^{q} a_{n-2, k-1} \geq 0$, $1 \leq k \leq n-q-1$. If $n=q, q+1$, then this statement is empty. In both cases, we complete the proof if we show that

$$
\Delta^{q} b_{n, 0} \geq 0 \text { and } \Delta^{q} b_{n, n-q} \geq 0 .
$$

Recall that, as in the proof of Lemma 2.2, we may assume that $f \in C^{q}[0,1]$. First, assume that $n \geq q+1$. Since $f(0)=0$,

$$
\begin{aligned}
\Delta^{1} b_{n, 0} & =a_{n-2,0}=\int_{0}^{1}(1-t)^{n-2} f(t) d t \\
& =\frac{1}{n-1} \int_{0}^{1}(1-t)^{n-1} f^{\prime}(t) d t=\frac{1}{n-1} \int_{0}^{1} p_{n-1,0}(t) f^{\prime}(t) d t
\end{aligned}
$$

and by $(2.5)$,

$$
\Delta^{1} b_{n, 1}=\Delta^{1} a_{n-2,0}=\frac{1}{n-1} \int_{0}^{1} p_{n-1,1}(t) f^{\prime}(t) d t .
$$

Hence, by (2.4),

$$
\Delta^{2} b_{n, 0}=-\frac{1}{n(n-1)} \int_{0}^{1} p_{n, 1}^{\prime}(t) f^{\prime}(t) d t=\frac{1}{n(n-1)} \int_{0}^{1} p_{n, 1}(t) f^{\prime \prime}(t) d t .
$$

Assuming, by induction, that

$$
\Delta^{q-1} b_{n, 0}=\frac{(n-2) !}{(n+q-3) !} \int_{0}^{1} p_{n+q-3, q-2}(t) f^{(q-1)}(t) d t,
$$

and noting that (2.3), for $q-1$, implies

$$
\Delta^{q-1} b_{n, 1}=\Delta^{q-1} a_{n-2,0}=\frac{(n-2) !}{(n+q-3) !} \int_{0}^{1} p_{n+q-3, q-1}(t) f^{(q-1)}(t) d t,
$$

we obtain, by (2.4) and integration by parts,

$$
\Delta^{q} b_{n, 0}=\frac{(n-2) !}{(n+q-2) !} \int_{0}^{1} p_{n+q-2, q-1}(t) f^{(q)}(t) d t \geq 0,
$$

that proves the left hand inequality in (2.7).

Similarly, since $f(1)=0$,

$$
\begin{aligned}
\Delta^{1} b_{n, n-1} & =-a_{n-2, n-2}=-\int_{0}^{1} t^{n-2} f(t) d t \\
& =\frac{1}{n-1} \int_{0}^{1} t^{n-1} f^{\prime}(t) d t=\frac{1}{n-1} \int_{0}^{1} p_{n-1, n-1}(t) f^{\prime}(t) d t,
\end{aligned}
$$


and by (2.5),

$$
\Delta^{1} b_{n, n-2}=\Delta^{1} a_{n-2, n-3}=\frac{1}{n-1} \int_{0}^{1} p_{n-1, n-2}(t) f^{\prime}(t) d t .
$$

Hence,

$$
\Delta^{2} b_{n, n-2}=-\frac{1}{n(n-1)} \int_{0}^{1} p_{n, n-1}^{\prime}(t) f^{\prime}(t) d t=\frac{1}{n(n-1)} \int_{0}^{1} p_{n, n-1}(t) f^{\prime \prime}(t) d t .
$$

Finally, again assuming, by induction, that

$$
\Delta^{q-1} b_{n, n-q+1}=\frac{(n-2) !}{(n+q-3) !} \int_{0}^{1} p_{n+q-3, n-1}(t) f^{(q-1)}(t) d t,
$$

we obtain as above,

$$
\Delta^{q} b_{n, n-q}=\frac{(n-2) !}{(n+q-2) !} \int_{0}^{1} p_{n+q-2, n-1}(t) f^{(q)}(t) d t \geq 0,
$$

that proves the right hand inequality in (2.7).

This completes the proof for $q \geq 2$ and $n \geq q+1$, so that we proceed to the case $n=q \geq 2$.

We begin with $n=q=2$, and observe that in this case,

$$
\left(U_{2} f\right)(x)=2 x(1-x) \int_{0}^{1} f(t) d t
$$

which is convex, since $f(t) \leq 0,0 \leq t \leq 1$.

If $n=q>2$, then we have to investigate $\Delta^{q} b_{n, 0}=\Delta^{q} b_{n, n-q}$, but both (2.9) and (2.11) are inapplicable. Thus we have to go back to (2.10) and (2.8).

We have

$$
\begin{aligned}
\Delta^{q} b_{q, 0} & =\Delta^{q-1} b_{q, 1}-\Delta^{q-1} b_{q, 0} \\
& =\frac{(q-2) !}{(2 q-3) !} \int_{0}^{1}\left(p_{2 q-3, q-1}(t)-p_{2 q-3, q-2}(t)\right) f^{(q-1)}(t) d t \\
& =-\frac{(q-2) !}{(2 q-2) !} \int_{0}^{1} p_{2 q-2, q-1}^{\prime}(t) f^{(q-1)}(t) d t \\
& =\frac{(q-2) !}{(2 q-2) !} \int_{0}^{1} p_{2 q-2, q-1}(t) f^{(q)}(t) d t \geq 0 .
\end{aligned}
$$

This completes the proof for all $n \geq q \geq 2$.

As for the case $q=1$, we observe that since $U_{n}$ preserves constants, we may assume that $f(0)=0$, so that, in turn,

$$
0 \leq \int_{0}^{1} f(t) d t \leq f(1) .
$$

First let $n>2$, and again denote $b_{n, 0}=0, b_{n, n}=0$ and $b_{n, k}=a_{n-2, k-1}$, $1 \leq k \leq n-1$. Then,

$$
\left(U_{n} f\right)(x)=f(1) x^{n}+(n-1) \sum_{k=0}^{n} p_{n, k}(x) b_{n, k},
$$


and

$$
\frac{d}{d x}\left(U_{n} f\right)(x)=n f(1) x^{n-1}+n(n-1) \sum_{k=0}^{n-1} p_{n-1, k}(x) \Delta^{1} b_{n, k} .
$$

Now, $\Delta^{1} b_{n, k} \geq 0,1 \leq k \leq n-2$, so we only need to check $\Delta^{1} b_{n, 0}$ and $\Delta^{1} b_{n, n-1}$.

To this end,

$$
\Delta^{1} b_{n, 0}=b_{n, 1}=a_{n-2,0}=\int_{0}^{1}(1-t)^{n-2} f(t) d t \geq 0
$$

since $f(t) \geq 0,0 \leq t \leq 1$.

On the other hand,

$\Delta^{1} b_{n, n-1}=-b_{n, n-1}=-a_{n-2, n-2}=-\int_{0}^{1} t^{n-2} f(t) d t \geq-\frac{f(1)}{n-1}$,

since $f$ is nondecreasing in $[0,1]$.

Hence,

$$
n f(1) x^{n-1}+n(n-1) p_{n-1, n-1}(x) \Delta^{1} b_{n, n-1} \geq 0,
$$

and it follows that $\left(U_{n} f\right)^{\prime}(x) \geq 0, x \in[0,1]$.

Finally, if $n=2$, then

$$
\begin{aligned}
\left(U_{2} f\right)(x) & =f(1) x^{2}+2 x(1-x) \int_{0}^{1} f(t) d t \\
& =\left(f(1)-\int_{0}^{1} f(t) d t\right) x^{2}+x(2-x) \int_{0}^{1} f(t) d t .
\end{aligned}
$$

Hence, by virtue of $(2.12), U_{2} f$ is nondecreasing in $[0,1]$. This completes the proof.

\section{The Szász-Durrmeyer operators}

The Favard-Mirakyan-Szász operators associated with a function $f$ defined in $[0, \infty)$, and such that $|f(x)| \leq C e^{A x}$ for some constants $C, A>0$, are defined by

$$
\left(S_{n} f\right)(x):=\sum_{k=0}^{\infty} s_{k}(n x) f\left(\frac{k}{n}\right), \quad x \in[0, \infty), \quad n \geq 1,
$$

where

$$
s_{k}(x):=e^{-x} \frac{x^{k}}{k !}, \quad 0 \leq x<\infty, \quad k \geq 0 .
$$

It is well-known that if, in addition, $f \in C[0, \infty)$, then the Favard-MirakyanSzász operators associated with $f$, approximate it uniformly on every compact subinterval of $[0, \infty)$. It is also known that they preserve $q$-monotonicity on $[0, \infty)$, which readily follows from the equation,

$$
\frac{d^{q}}{d x^{q}}\left(S_{n} f\right)(x)=n^{q} \sum_{k=0}^{\infty} s_{k}(n x) \Delta^{q} f\left(\frac{k}{n}\right) .
$$


In 1985, Mazhar and Totik [13] defined the Szász-Durrmeyer variant for continuous functions of the above type, by

$$
\left(L_{n} f\right)(x):=n \sum_{k=0}^{\infty} s_{k}(n x) \int_{0}^{\infty} s_{k}(n t) f(t) d t, \quad x \in[0, \infty), \quad n>A .
$$

We will prove the following.

Theorem 3.1. Given $q \geq 1$, suppose that $f \in C[0, \infty)$ is $q$-monotone and there exist constants $C, A \geq 0$ such that $|f(x)| \leq C e^{A x}, 0 \leq x<\infty$. Then $L_{n} f, n>A$, is q-monotone in $[0, \infty)$.

A weaker result but easier to prove, and which goes along the lines of the proof of Lemma 2.2 is

Theorem 3.2. Given $q \geq 1$, suppose that $f \in C^{q}[0, \infty)$ is $q$-monotone and there exist constants $C, A \geq 0$ such that $\left|f^{(i)}(x)\right| \leq C e^{A x}, 0 \leq i \leq q-1$, $0 \leq x<\infty$. Then $L_{n} f, n>A$, is q-monotone in $[0, \infty)$.

We may characterize $q$-monotone functions in $[0, \infty)$, with an analog of Corollary 2.3. For this we need the notation

$$
a_{n, k}:=\int_{0}^{\infty} s_{k}(n t) f(t) d t
$$

Corollary 3.3. Let $q \geq 1$ and assume that $f \in C[0, \infty)$ is such that $|f(x)| \leq$ $C e^{A x}, 0 \leq x<\infty$, for some $C, A \geq 0$. Then, $f$ is q-monotone in $[0, \infty)$, if and only if,

$$
\Delta^{q} a_{n, k} \geq 0, \quad n>A \text { and } k \geq 0 .
$$

Proof. From the proof of Theorem 3.1 we conclude that if $f$ is $q$-monotone, then (3.3) is valid. Conversely, we observe from the proof of Theorem 3.1 that (3.3) implies that $L_{n} f, n>A$ is $q$-monotone. Since $\left(L_{n} f\right)(x) \rightarrow f(x)$, $x \in[0, \infty)$, it follows that $f$ is $q$-monotone in $[0, \infty)$.

We begin with the proof of Theorem 3.2.

Proof of Theorem 3.2. Similar to (3.1), we obtain,

$$
\frac{d^{q}}{d x^{q}}\left(L_{n} f\right)(x)=n^{q+1} e^{-n x} \sum_{k=0}^{\infty} \frac{(n x)^{k}}{k !} \Delta^{q} a_{n, k}, \quad n>A,
$$

where $a_{n, k}$ are defined in (3.2).

Hence, our proof will be complete if we prove that $\Delta^{q} a_{n, k} \geq 0, k=$ $0,1,2 \ldots, n>A$.

To this end, since

$$
s_{k+1}^{\prime}(n t)=-n\left(s_{k+1}(n t)-s_{k}(n t)\right),
$$

we get

$$
\Delta^{1} a_{n, k}=-\frac{1}{n} \int_{0}^{\infty} s_{k+1}^{\prime}(n t) f(t) d t=\frac{1}{n} \int_{0}^{\infty} s_{k+1}(n t) f^{\prime}(t) d t .
$$


Proceeding by induction we obtain

$$
\Delta^{q} a_{n, k}=\frac{1}{n^{q}} \int_{0}^{\infty} s_{k+q}(n t) f^{(q)}(t) d t \geq 0 .
$$

Note that due to the integration by parts, we do not have to assume growth conditions on $f^{(q)}$, for the integral to converge. This completes the proof.

Proof of Theorem 3.1. As above, we need to prove that $\Delta^{q} a_{n, k} \geq 0$. We observe that

$$
\begin{aligned}
\Delta^{q} a_{n, k} & =\int_{0}^{\infty} e^{-n t} \sum_{j=0}^{q}(-1)^{q-j}\left(\begin{array}{l}
q \\
j
\end{array}\right) \frac{(n t)^{k+j}}{(k+j) !} f(t) d t \\
& =\frac{(-1)^{q}}{n^{q}} \int_{0}^{\infty}\left(e^{-n t} \frac{(n t)^{k+q}}{(k+q) !}\right)^{(q)} f(t) d t \\
& =\frac{(-1)^{q}}{n^{q}} \int_{0}^{\infty} s_{k+q}^{(q)}(n t) f(t) d t .
\end{aligned}
$$

Denote $s_{k+q}(n t)=: g(t)$ and let $\Delta_{h}^{q} g(t):=\sum_{j=0}^{q}(-1)^{q-j}\left(\begin{array}{l}q \\ j\end{array}\right) g(t+j h)$. Rewrite

$$
\begin{aligned}
\Delta^{q} a_{n, k}= & \frac{(-1)^{q}}{n^{q} h^{q}} \int_{0}^{\infty} \Delta_{h}^{q} g(t) f(t) d t \\
& +\left(\frac{(-1)^{q}}{n^{q}} \int_{0}^{\infty} g^{(q)}(t) f(t) d t-\frac{(-1)^{q}}{n^{q} h^{q}} \int_{0}^{\infty} \Delta_{h}^{q} g(t) f(t) d t\right) \\
= & : \frac{(-1)^{q}}{n^{q} h^{q}} \int_{0}^{\infty} \Delta_{h}^{q} g(t) f(t) d t+R_{1} .
\end{aligned}
$$

We will prove that $R_{1} \rightarrow 0$, as $h \rightarrow 0$. To this end, note that for some $\xi \in(t, t+q h)$,

$$
\begin{aligned}
\left|h^{-q} \Delta_{h}^{q} g(t)-g^{(q)}(t)\right| & =\left|g^{(q)}(\xi)-g^{(q)}(t)\right| \leq|\xi-t| \max _{t \leq u \leq t+q h}\left|g^{(q+1)}(u)\right| \\
& \leq q h \max _{t \leq u \leq t+q h}\left|g^{(q+1)}(u)\right| .
\end{aligned}
$$

Hence,

$$
\begin{aligned}
\left|R_{1}\right| & \leq q h n^{-q} \int_{0}^{\infty}\left[\max _{t \leq u \leq t+q h}\left|g^{(q+1)}(u)\right|\right]|f(t)| d t \\
& \leq \operatorname{Cqhn} \sum_{j=0}^{q+1}\left(\begin{array}{c}
q+1 \\
j
\end{array}\right) \int_{0}^{\infty}\left[\max _{t \leq u \leq t+q h}\left(e^{-n u} \frac{(n u)^{k+j}}{(k+j) !}\right)\right] e^{A t} d t .
\end{aligned}
$$

Thus, it suffices to prove that for $m=0,1,2, \ldots$,

$$
\lim _{h \rightarrow 0} h \int_{0}^{\infty} \max _{t \leq u \leq t+q h}\left(e^{-n u}(n u)^{m}\right) e^{A t} d t=0 .
$$

Since $e^{-n u}(n u)^{m} \leq m$ !, and it is increasing in $[0, m / n]$ and decreasing in $[m / n, \infty)$, we conclude that

$$
\int_{0}^{\infty} \max _{t \leq u \leq t+q h}\left(e^{-n u}(n u)^{m}\right) e^{A t} d t \leq m ! \int_{0}^{m / n} e^{A t} d t+\int_{m / n}^{\infty} e^{-n t}(n t)^{m} e^{A t} d t<\infty .
$$


Hence, $R_{1}=O(h)$, as $h \rightarrow 0$.

Now,

$$
\begin{aligned}
\int_{0}^{\infty} \Delta_{h}^{q} g(t) f(t) d t= & \sum_{j=0}^{q}(-1)^{q-j}\left(\begin{array}{l}
q \\
j
\end{array}\right) \int_{0}^{\infty} g(t+j h) f(t) d t \\
= & \sum_{j=0}^{q}(-1)^{q-j}\left(\begin{array}{l}
q \\
j
\end{array}\right) \int_{j h}^{\infty} g(t) f(t-j h) d t \\
= & \sum_{j=0}^{q}(-1)^{j}\left(\begin{array}{l}
q \\
j
\end{array}\right) \int_{(q-j) h}^{\infty} g(t) f(t-q h+j h) d t \\
= & (-1)^{q} \int_{q h}^{\infty} g(t) \Delta_{h}^{q} f(t-q h) d t \\
& +\sum_{j=0}^{q}(-1)^{j}\left(\begin{array}{l}
q \\
j
\end{array}\right) \int_{(q-j) h}^{q h} g(t) f(t-q h+j h) d t \\
= & :(-1)^{q} \int_{q h}^{\infty} g(t) \Delta_{h}^{q} f(t-q h) d t+R_{2} .
\end{aligned}
$$

Now

$\left|R_{2}\right| \leq C\left(2^{q}-1\right) e^{q A h} \int_{0}^{q h} \frac{(n t)^{k+q}}{(k+q) !} d t=C\left(2^{q}-1\right) e^{q A h} \frac{n^{k+q}}{(k+q+1) !}(q h)^{k+q+1}$.

Finally, by (3.5), we have

$$
\Delta^{q} a_{n, k}=\frac{1}{n^{q} h^{q}} \int_{q h}^{\infty} e^{-n t} \frac{(n t)^{k+q}}{(k+q) !} \Delta_{h}^{q} f(t-q h) d t+R
$$

where $R:=R_{1}+\frac{1}{(n h)^{q}} R_{2}=O(h)$, as $h \rightarrow 0$.

If $f$ is $q$-monotone, then we get for all $h>0$ that

$$
\Delta^{q} a_{n, k} \geq R .
$$

Hence, as the left hand side is independent of $h$, we conclude that $\Delta^{q} a_{n, k} \geq 0$, and the proof is complete.

\section{The Baskakov-Durrmeyer operators}

The Baskakov operators are defined for a function $f$ of polynomial growth on $[0, \infty)$, by

$$
\left(M_{n} f\right)(x):=\sum_{k=0}^{\infty}\left(\begin{array}{c}
n+k-1 \\
k
\end{array}\right) x^{k}(1+x)^{-n-k} f\left(\frac{k}{n}\right), \quad x \in[0, \infty), \quad n \geq 1 .
$$

It is well-known that if, in addition, $f \in C[0, \infty)$, then the Baskakov operators associated with $f$, approximate it uniformly on every compact subinterval of 
$[0, \infty)$. It is also known that they preserve $q$-monotonicity on $[0, \infty)$, which readily follows from the equation,

$$
\begin{aligned}
& \frac{d^{q}}{d x^{q}}\left(M_{n} f\right)(x) \\
& \quad=\frac{(n+q-1) !}{(n-1) !} \sum_{k=0}^{\infty}\left(\begin{array}{c}
n+q+k-1 \\
k
\end{array}\right) x^{k}(1+x)^{-n-q-k} \Delta^{q} f\left(\frac{k}{n}\right) .
\end{aligned}
$$

Denoting

$$
m_{n, k}(x):=\left(\begin{array}{c}
n+k-1 \\
k
\end{array}\right) x^{k}(1+x)^{-n-k}, \quad x \in[0, \infty), \quad n \geq 1, k \geq 0,
$$

Sahai and Prasad [15] have defined the Baskakov-Durrmeyer variant of the above operators, for $f$ such that $|f(x)| \leq C(x+1)^{A}, C, A \geq 0$, by

$$
\left(V_{n} f\right)(x):=(n-1) \sum_{k=0}^{\infty} m_{n, k}(x) \int_{0}^{\infty} m_{n, k}(t) f(t) d t, \quad x \in[0, \infty), \quad n>A+1 .
$$

Remark 4.1. In [15] the definition of the operators is only for integrable functions while one may define the operators for large enough $n$, for functions of polynomial growth. On the other hand, we prefer to assume that the functions are continuous in $[0, \infty)$, which, for $q \geq 2$, amounts to assuming continuity at $x=0$. This way we are guaranteed the approximation on compact subintervals of $[0, \infty)$.

For the Baskakov-Durrmeyer operators we have two results analogous to Theorems 3.1 and 3.2. Namely,

Theorem 4.2. Given $q \geq 1$, suppose that $f \in C[0, \infty)$ is $q$-monotone and there exist constants $C, A \geq 0$ such that $|f(x)| \leq C(x+1)^{A}, 0 \leq x<\infty$. Then $V_{n} f, n \geq 1, n>A+1$, is q-monotone in $[0, \infty)$.

Again, a weaker result, which is easier to prove, is

Theorem 4.3. Given $q \geq 1$, suppose that $f \in C^{q}[0, \infty)$ is $q$-monotone and there exist constants $C, A \geq 0$ such that $\left|f^{(i)}(x)\right| \leq C(x+1)^{A}, 0 \leq i \leq q-1$, $0 \leq x<\infty$. Then $V_{n} f, n>A+q$, is q-monotone in $[0, \infty)$.

Yet another characterization of continuous $q$-monotone functions in $[0, \infty)$ is the next corollary that is proved exactly as Corollary 3.3, thus we omit the proof. Denote

$$
a_{n, k}:=\int_{0}^{\infty} m_{n, k}(t) f(t) d t
$$

Corollary 4.4. Let $q \geq 1$ and assume that $f \in C[0, \infty)$ is such that $|f(x)| \leq$ $C(x+1)^{A}, 0 \leq x<\infty$, for some $C, A \geq 0$. Then, $f$ is $q$-monotone in $[0, \infty)$, if and only if, (3.3) is valid, but this time, for $n>A+1$.

We begin with the proof of Theorem 4.3. 
Proof of Theorem 4.3. The proof follows the lines of the proof of Theorem 3.1. Similar to (4.1), we obtain,

$$
\frac{d^{q}}{d x^{q}}\left(V_{n} f\right)(x)=\frac{(n+q-1) !}{(n-2) !} \sum_{k=0}^{\infty} m_{n+q, k}(x) \Delta^{q} a_{n, k} .
$$

Thus, we will conclude if we show that $\Delta^{q} a_{n, k} \geq 0$. To this end, we observe that

$$
m_{n, k+1}^{\prime}(x)=-n\left(m_{n+1, k+1}(x)-m_{n+1, k}(x)\right) .
$$

Hence, for $n \geq 2$,

$$
\Delta^{1} a_{n, k}=-\frac{1}{n-1} \int_{0}^{\infty} m_{n-1, k+1}^{\prime}(t) f(t) d t=\frac{1}{n-1} \int_{0}^{\infty} m_{n-1, k+1}(t) f^{\prime}(t) d t .
$$

and by induction, for $n \geq q+1$,

$$
\Delta^{q} a_{n, k}=\frac{(n-q-1) !}{(n-1) !} \int_{0}^{\infty} m_{n-q, k+q}(t) f^{(q)}(t) d t \geq 0 .
$$

Again, note that we do not need to assume growth conditions on $f^{(q)}$, for the integral to converge. This completes the proof.

Proof of Theorem 4.2. The proof is along the lines of the proof of Theorem 3.1 with the obvious changes. Similar to (3.5), we may write

$$
\Delta^{q} a_{n, k}=(-1)^{q} \frac{(n-q-1) !}{(n-1) ! h^{q}} \int_{0}^{\infty} \Delta_{h}^{q} g(t) f(t) d t+R_{1},
$$

where now $g(t):=m_{n-q, k+q}(t)$, and

$$
\left|R_{1}\right| \leq q h \frac{(n-q-1) !}{(n-1) !} \int_{0}^{\infty}\left[\max _{t \leq u \leq t+q h}\left|g^{(q+1)}(u)\right|\right]|f(t)| d t .
$$

Since

$$
m_{n-q, k+q}^{(q+1)}(u)=(-1)^{q+1} \frac{n !}{(n-q-1) !} \sum_{i=0}^{q+1}(-1)^{i}\left(\begin{array}{c}
q+1 \\
i
\end{array}\right) m_{n+1, k+i-1}(u),
$$

(where $m_{n+1,-1}(u) \equiv 0$ ), all we have to do is to prove that for any pair $(n, k)$,

$$
\lim _{h \rightarrow 0} h \int_{0}^{\infty}\left[\max _{t \leq u \leq t+q h} m_{n+1, k}(u)\right](t+1)^{A} d t=0 .
$$

Now, $m_{n+1, k}(u) \leq 1$, and it is increasing in $[0, k /(n+1)]$ and decreasing in $[k /(n+1), \infty)$. Thus,

$$
\begin{aligned}
& \int_{0}^{\infty}\left[\max _{t \leq u \leq t+q h} m_{n+1, k}(u)\right](t+1)^{A} d t \\
& \quad \leq \int_{0}^{k /(n+1)}(t+1)^{A} d t+\left(\begin{array}{c}
n+k \\
k
\end{array}\right) \int_{k /(n+1)}^{\infty} t^{k}(t+1)^{A}(1+t)^{-n-k-1} d t<\infty .
\end{aligned}
$$

Hence, $R_{1}=O(h)$, as $h \rightarrow 0$.

By virtue of (3.6), we may write

$$
\int_{0}^{\infty} \Delta_{h}^{q} g(t) f(t) d t=(-1)^{q} \int_{q h}^{\infty} g(t) \Delta_{h}^{q} f(t-q h) d t+R_{2} .
$$


Again,

$$
\left|R_{2}\right| \leq C\left(\begin{array}{c}
n+k-1 \\
k+q
\end{array}\right)\left(2^{q}-1\right)(q h+1)^{A} \frac{(q h)^{k+q+1}}{k+q+1} .
$$

Therefore, as before,

$$
\Delta^{q} a_{n, k}=\frac{(n-q-1) !}{(n-1) ! h^{q}} \int_{q h}^{\infty} m_{n-q, k+q}(t) \Delta_{h}^{q} f(t-q h) d t+R,
$$

where $R:=R_{1}+\frac{(n-q-1) !}{(n-1) ! h^{q}} R_{2}=O(h)$, as $h \rightarrow 0$.

If $f$ is $q$-monotone, then

$$
\Delta^{q} a_{n, k} \geq R .
$$

Hence, as the left hand side is independent of $h$, we conclude that $\Delta^{q} a_{n, k} \geq 0$, and the proof is complete.

\section{Concluding comments}

In [1] the authors prove a $q$ th version of Raşa inequality for the ordinary Bernstein, Favard-Mirakyan-Szász and Baskakov operators. We state the analogous results for the Durrmeyer variants of those operators. One readily observes that the proofs depend only on the appropriate $\Delta^{q}$ differences being nonnegative. Hence, one may follow those in [1] verbatim, so we skip them here. We have,

Theorem 5.1. Let $q, n \in \mathbb{N}$ and assume that $f \in C[0,1]$ is q-monotone. Then for all $x, y \in[0,1]$,

$$
\begin{aligned}
& \operatorname{sgn}(x-y)^{q} \sum_{\nu_{1}, \ldots, \nu_{q}=0}^{n} \sum_{j=0}^{q}(-1)^{q-j}\left(\begin{array}{l}
q \\
j
\end{array}\right)\left(\prod_{i=1}^{j} p_{n, \nu_{i}}(x)\right)\left(\prod_{i=j+1}^{q} p_{n, \nu_{i}}(y)\right) \\
& \times \int_{0}^{\infty} p_{q n, \nu_{1}+\cdots+\nu_{q}}(t) f(t) d t \geq 0 .
\end{aligned}
$$

Theorem 5.2. Let $q \geq 1$ and assume that $f \in C[0, \infty)$ is q-monotone and is such that $|f(x)| \leq C e^{A x}, x \in[0, \infty)$. Then for all $x, y \in[0, \infty)$ and each $n>A / q$,

$$
\begin{aligned}
& \operatorname{sgn}(x-y)^{q} \sum_{\nu_{1}, \ldots, \nu_{q}=0}^{n} \sum_{j=0}^{q}(-1)^{q-j}\left(\begin{array}{l}
q \\
j
\end{array}\right) e^{-n(j x+(q-j) y)} \frac{(n x)^{\nu_{1}+\cdots+\nu_{j}}(n y)^{\nu_{j+1}+\cdots+\nu_{q}}}{\nu_{1} ! \cdots \nu_{q} !} \\
& \quad \times \int_{0}^{\infty} e^{-q n t} \frac{(n t)^{\nu_{1}+\cdots+\nu_{q}}}{\left(\nu_{1}+\cdots+\nu_{q}\right) !} f(t) d t \geq 0 .
\end{aligned}
$$

And finally, 
Theorem 5.3. Let $q \geq 1$ and assume that $f \in C[0, \infty)$ is $q$-monotone and is such that $|f(x)| \leq C(x+1)^{A}, x \in[0, \infty)$. Then for all $x, y \in[0, \infty)$ and each $n>(A+1) / q$,

$$
\begin{aligned}
& \operatorname{sgn}(x-y)^{q} \sum_{\nu_{1}, \ldots, \nu_{q}=0}^{n} \sum_{j=0}^{q}(-1)^{q-j}\left(\begin{array}{l}
q \\
j
\end{array}\right)\left(\prod_{i=1}^{j} m_{n, \nu_{i}}(x)\right)\left(\prod_{i=j+1}^{q} m_{n, \nu_{i}}(y)\right) \\
& \times \int_{0}^{\infty} m_{q n, \nu_{1}+\cdots+\nu_{q}}(t) f(t) d t \geq 0 .
\end{aligned}
$$

\section{Acknowledgements}

The authors are grateful to the anonymous referees, who have rigorously read the manuscript, for their corrections and valuable comments.

Funding Open Access funding enabled and organized by Projekt DEAL.

Open Access. This article is licensed under a Creative Commons Attribution 4.0 International License, which permits use, sharing, adaptation, distribution and reproduction in any medium or format, as long as you give appropriate credit to the original author(s) and the source, provide a link to the Creative Commons licence, and indicate if changes were made. The images or other third party material in this article are included in the article's Creative Commons licence, unless indicated otherwise in a credit line to the material. If material is not included in the article's Creative Commons licence and your intended use is not permitted by statutory regulation or exceeds the permitted use, you will need to obtain permission directly from the copyright holder. To view a copy of this licence, visit http:// creativecommons.org/licenses/by/4.0/.

Publisher's Note Springer Nature remains neutral with regard to jurisdictional claims in published maps and institutional affiliations.

\section{References}

[1] Abel, U., Leviatan, D.: An extension of Raşa's conjecture to q-monotone functions. Results Math. 75, 180-192 (2020)

[2] Adell, J.A., de la Cal, J.: Bernstein-Durrmeyer operators. Comput. Math. Appl. 30(3-6), 1-14 (1995)

[3] Attalienti, A., Raşa, I.: Total positivity: an application to positive linear operators and their limiting semigroups. Rev. Anal. Numér. Théor. Approx. 36(1), 51-66 (2007)

[4] Derriennic, M.M.: Sur l'approximation de functions intégrables sur [0,1] par des polynômes de Bernstein modifies. J. Approx. Theory 31, 325-343 (1981)

[5] Ditzian, Z., Ivanov, K.: Bernstein-type operators and their derivatives. J. Approx. Theory 56, 72-90 (1989)

[6] Durrmeyer, J.L.: Une formule d'inversion de la transformée de Laplace: Applications à la theorie des moments, Thése de 3e cycle, Paris (1967)

[7] Gonska, H., Kacsó, D., Raşa, I.: The genuine Bernstein-Durrmeyer operators revisited. Results Math. 62, 295-310 (2012) 
[8] Goodman, T.N.T., Sharma, A.: A modified Bernstein-Schoenberg operator, In: Sendov, Bl., et al. (eds.) Proceedings of the Conference on Constructive Theory of Functions, Varna 1987, pp. 166-173. Publ. House, Bulg. Acad. of Sci., Sofia, Bulg (1988)

[9] Heilmann, M., Raşa, I.: A nice representation for a link between Baskakov- and Szász-Mirakjan-Durrmeyer operators and their Kantorovich variants. Results Math. 74(9), 1-12 (2019)

[10] Karlin, S.: Total positivity, 1. Stanford University Press, Stanford (1968)

[11] Lorentz, G.G.: Bernstein Polynomials, Mathematical Expositions, 8. University of Toronto Press, Toronto (1953)

[12] Lupaş, A.: Die Folge der Betaoperatoren, Dissertation, Universität Stuttgart (1972)

[13] Mazhar, S.M., Totik, V.: Approximation by modified Szász operators. Acta Sci. Math. (Szeged) 49, 257-269 (1985)

[14] Păltănea, R.: Simultaneous approximation by a class of Szász-Mirakjan operators. J. Appl. Funct. Anal. 9, 356-368 (2014)

[15] Sahai, A., Prasad, G.: On simultaneous approximation by modified Lupaş operators. J. Approx. Theory 45, 122-128 (1985)

Ulrich Abel

Fachbereich MND

Technische Hochschule Mittelhessen

Wilhelm-Leuschner-Straße 13

61169 Friedberg

Germany

e-mail: Ulrich.Abel@mnd.thm.de

Dany Leviatan

Raymond and Beverly Sackler School of Mathematical Sciences

Tel Aviv University

Tel Aviv 6139001

Israel

e-mail: leviatan@tauex.tau.ac.il

Ioan Raşa

Department of mathematics

Technical University of Cluj-Napoca: Universitatea Tehnica din Cluj-Napoca

Str. Memorandumului nr. 28

400114 Cluj-Napoca

Romania

e-mail: Ioan.Rasa@math.utcluj.ro

Received: February 1, 2021.

Revised: May 19, 2021.

Accepted: June 9, 2021. 